\title{
ON EFFECTIVE EQUIDISTRIBUTION OF EXPANDING TRANSLATES OF CERTAIN ORBITS IN THE SPACE OF LATTICES
}

\author{
D. Y. Kleinbock and G. A. Margulis
}

\begin{abstract}
We prove an effective version of a result obtained earlier by Kleinbock and Weiss $[\mathrm{KW}]$ on equidistribution of expanding translates of orbits of horospherical subgroups in the space of lattices.
\end{abstract}

\section{INTRODUCTION}

The motivation for this work is a result obtained recently in $[\mathrm{KW}]$. Fix $m, n \in \mathbb{N}$, set $k=m+n$ and let

$$
G=\mathrm{SL}_{k}(\mathbb{R}), \Gamma=\mathrm{SL}_{k}(\mathbb{Z}), u_{Y}=\left(\begin{array}{cc}
I_{m} & Y \\
0 & I_{n}
\end{array}\right), H=\left\{u_{Y} \mid Y \in M_{m, n}\right\}
$$

where $M_{m, n}$ stands for the space of $m \times n$ matrices with real entries. Then $H$ is a unipotent abelian subgroup of $G$ which is expanding horospherical with respect to

$$
g_{t}=\operatorname{diag}\left(e^{t / m}, \ldots, e^{t / m}, e^{-t / n}, \ldots, e^{t / n}\right), \quad t>0 .
$$

The latter, by definition, means that the Lie algebra of $H$ is the span of eigenspaces of $\operatorname{Ad}\left(g_{t}\right), t>0$, with eigenvalues bigger than 1 in absolute value.

The space $X \stackrel{\text { def }}{=} G / \Gamma$ can be identified with the space of unimodular lattices in $\mathbb{R}^{k}$, on which $G$ acts by left translations. Denote by $\pi$ the natural projection $G \rightarrow X, g \mapsto g \Gamma$, and for any $z \in X$ let $\pi_{z}: G \rightarrow X$ be defined by $\pi_{z}(g)=g z$. Also denote by $\bar{\mu}$ the $G$-invariant probability measure on $X$ and by $\mu$ the Haar measure on $G$ such that $\pi_{*} \mu=\bar{\mu}$. Fix a Haar measure $\nu$ on $H$. Note that the $H$-orbit foliation is unstable with respect to the action of $g_{t}, t>0$. It is well known that for any Borel probability measure $\nu^{\prime}$ on $H$ absolutely continuous with respect to $\nu$ and for any $z \in X, g_{t}$-translates of $\left(\pi_{z}\right)_{*} \nu^{\prime}$ become equidistributed, that is, weak-* converge to $\bar{\mu}$ as $t \rightarrow \infty$. An effective version of this statement was obtained in [KM1, Proposition 2.4.8]. In order to state that result, it will be convenient to introduce the following notation: for $f \in L^{1}(H, \nu)$, a bounded continuous function $\psi$ on $X, z \in X$ and $g \in G$ define

$$
I_{f, \psi}(g, z) \stackrel{\text { def }}{=} \int_{H} f(h) \psi\left(g_{t} h z\right) d \nu(h) .
$$

1991 Mathematics Subject Classification. 37A17; 37A25. 
In other words, $I_{f, \psi}(g, z)$ is the result of evaluation of the $g$-translate of $\left(\pi_{z}\right)_{*} \nu^{\prime}$ at $\psi$, where $d \nu^{\prime}=f d \nu$. Then equidistribution of $g_{t}$-translates of $\left(\pi_{z}\right)_{*} \nu^{\prime}$ amounts to the convergence of $I_{f, \psi}\left(g_{t}, z\right)$ to $\int_{H} f \cdot \int_{X} \psi$ as $t \rightarrow \infty$ (unless it causes confusion, we will omit measures in the integration notation for the sake of brevity).

The following is a slightly simplified form of [KM1, Proposition 2.4.8]:

Theorem 1.1. There exists $\gamma>0$ such that for any $f \in C_{\text {comp }}^{\infty}(H), \psi \in C_{\text {comp }}^{\infty}(X)$ and for any compact subset $L$ of $X$ there exists a constant $C=C(f, \psi, L)$ such that for all $z \in L$ and any $t \geq 0$

$$
\left|I_{f, \psi}\left(g_{t}, z\right)-\int_{H} f \int_{X} \psi\right| \leq C e^{-\gamma t} .
$$

The proof used the exponential decay of correlations of the $G$-action on $X$ (called 'condition (EM)' in [KM1]). See $\S 2$ for more detail.

Motivated by some questions in simultaneous Diophantine approximation, the first named author and Barak Weiss considered translates of $H$-orbits on $X$ by diagonal elements of $G$ other than $g_{t}$. Specifically, following $[\mathrm{KW}]$, let us denote by $\mathfrak{a}^{+}$the set of $k$-tuples $\mathbf{t}=\left(t_{1}, \ldots, t_{k}\right) \in \mathbb{R}^{k}$ such that

$$
t_{1}, \ldots, t_{k}>0 \text { and } \sum_{i=1}^{m} t_{i}=\sum_{j=1}^{n} t_{m+j}
$$

and for $\mathbf{t} \in \mathfrak{a}_{+}$define

$$
g_{\mathbf{t}} \stackrel{\text { def }}{=} \operatorname{diag}\left(e^{t_{1}}, \ldots, e^{t_{m}}, e^{-t_{m+1}}, \ldots, e^{-t_{k}}\right) \in G
$$

and

$$
\lfloor\mathbf{t}\rfloor \stackrel{\text { def }}{=} \min _{i=1, \ldots, k} t_{i}
$$

(the latter, roughly speaking, measures the distance between $\mathbf{t}$ and the walls of the cone $\mathfrak{a}^{+} \subset \mathbb{R}^{k}$ ).

The theorem below is a reformulation of [KW, Theorem 2.2]:

Theorem 1.2. For any $f \in L^{1}(H, \nu)$, any continuous compactly supported $\psi$ : $X \rightarrow \mathbb{R}$, any compact subset $L$ of $X$ and any $\varepsilon>0$ there exists $T>0$ such that

$$
\left|I_{f, \psi}\left(g_{\mathbf{t}}, z\right)-\int_{H} f \int_{X} \psi\right|<\varepsilon
$$

for all $z \in L$ and $\mathbf{t} \in \mathfrak{a}^{+},\lfloor\mathbf{t}\rfloor \geq T$.

That is, $g_{\mathbf{t}}$-translates of $H$-orbits become equidistributed as $\lfloor\mathbf{t}\rfloor \rightarrow \infty$ uniformly in $z$ when the latter is restricted to compact subsets of $X$. The proof relies on S. G. Dani's classification of measures invariant under horospherical subgroups and the so-called 'linearization method'. The purpose of the present paper is to prove an effective version of the above theorem: 
Theorem 1.3. There exists $\tilde{\gamma}>0$ such that for any $f \in C_{\text {comp }}^{\infty}(H), \psi \in C_{\text {comp }}^{\infty}(X)$ and for any compact $L \subset X$ there exists $\tilde{C}=\tilde{C}(f, \psi, L)$ such that for all $z \in L$ and all $\mathbf{t} \in \mathfrak{a}^{+}$

$$
\left|I_{f, \psi}\left(g_{\mathbf{t}}, z\right)-\int_{H} f \int_{X} \psi\right| \leq \tilde{C} e^{-\tilde{\gamma}\lfloor\mathbf{t}\rfloor} .
$$

Note that the above statement follows from Theorem 1.1 when $k=2$, that is, $G=\mathrm{SL}_{2}(\mathbb{R})$, but is new for $k>2$. The proof uses the 'exponential mixing' approach of [KM1, KM3] together with effective nondivergence estimates obtained in [KM2]. We will describe these two parts in $\S \S 2$ and 3 respectively, and then proceed with the proof of Theorem 1.3 in $\S 4$. We remark that the method of proof readily extends to the set-up more general than (1.1). Note also that, as observed by N. Shah in [S, Remark 1.0.2], Theorem 1.3 can be used to strengthen one of the main results of $[\mathrm{KW}]$, that is, [KW, Theorem 1.4], which constitutes a Diophantine application of Theorem 1.2.

Acknowledgements. The authors are grateful to the Fields Institute for Research in Mathematical Sciences (Toronto, Canada), where this project has commenced, and to the referee for useful remarks. The work of the first named author was supported in part by NSF Grants DMS-0239463 and DMS-0801064, and that of the second author by NSF Grants DMS-0244406 and DMS-0801195.

\section{EXPONENTIAL MIXING AND $g_{t}$-TRANSLATES}

Notation: We will fix a right-invariant metric 'dist' on $G$, giving rise to the corresponding metric on $X$. $B(x, r)$ will stand for an open ball of radius $r$ centered at $x$. If a metric space is $G$ or its subgroups, we will abbreviate $B(e, r)$ to $B(r)$. When necessary, we will use subscripts denoting the ambient metric spaces. $\|\cdot\|_{\ell}$ and $\|\cdot\|_{C^{\ell}}$ will stand for the $(2, \ell)$-Sobolev and $C^{l}$ norms respectively. We define

$$
W^{2, \infty}(X)=\left\{\psi \in C^{\infty}(X):\|\psi\|_{\ell}<\infty \forall \ell \in \mathbb{N}\right\}
$$

clearly $C_{\text {comp }}^{\infty}(X) \subset W^{2, \infty}(X)$. In fact, $W^{2, \infty}(X)$ coincides with the set of functions $\psi \in C^{\infty}(X)$ such that $D \psi \in L^{2}(X)$ for any $D$ from the universal enveloping algebra of $\operatorname{Lie}(G)$. We let $\langle\cdot, \cdot\rangle$ stand for the inner product in $L^{2}(X)$. We also denote by $\|\psi\|_{\text {Lip }}$ the Lipschitz constant of a function $\psi$ on $X$,

$$
\|\psi\|_{\text {Lip }} \stackrel{\text { def }}{=} \sup _{x, y \in X, x \neq y} \frac{|\psi(x)-\psi(y)|}{\operatorname{dist}(x, y)}
$$

and let $\operatorname{Lip}(X) \stackrel{\text { def }}{=}\left\{\psi:\|\psi\|_{\text {Lip }}<\infty\right\}$.

The following property of the $G$-action on $X$ is deduced in [KM3] from the spectral gap on $L^{2}(X)$ :

Theorem 2.1 [KM3, Corollary 3.5]. There exist $\gamma>0$ and $\ell \in \mathbb{N}$ such that for any two functions $\varphi, \psi \in W^{2, \infty}(X)$ and for any $t \geq 0$ one has

$$
\left|\langle g \varphi, \psi\rangle-\int_{X} \varphi \int_{X} \psi\right| \ll\|\varphi\|_{\ell}\|\psi\|_{\ell} \cdot e^{-\gamma \operatorname{dist}(g, e)} .
$$


Here and hereafter the implicit constants in $\ll$ depend only on the dimensions of the corresponding spaces and the choices of the metric. Taking $g=g_{t}$ as in (1.2), it follows that

$$
\left|\left\langle g_{t} \varphi, \psi\right\rangle-\int_{X} \varphi \int_{X} \psi\right| \ll\|\varphi\|_{\ell}\|\psi\|_{\ell} \cdot e^{-\gamma t}
$$

An estimate analogous to (2.1) was used in [KM1] to derive Theorem 1.1. In this section we apply Theorem 2.1 to prove a statement similar to Theorem 1.1, providing some information as to how $C$ in (1.3) depends on $f$ and $L$. The argument follows the lines of the proof in [KM1]; in fact, the statement below is basically an intermediate step in the proof of [KM1, Proposition 2.4.8]. However we have decided to include details for the sake of making this paper self-contained.

To pass from $\left\langle g_{t} \varphi, \psi\right\rangle$ to $I_{f, \psi}\left(g_{t}, z\right)$, we need to thicken $f$ into a suitable function $\varphi$ on $X$. To explain this process, we need to introduce some more notation. Let

$$
H^{-}=\left\{\left(\begin{array}{cc}
I_{m} & 0 \\
Y & I_{n}
\end{array}\right) \mid Y \in M_{n, m}\right\}
$$

and

$$
H^{0}=\left\{\left(\begin{array}{cc}
A & 0 \\
0 & B
\end{array}\right) \mid A \in \mathrm{GL}_{m}(\mathbb{R}), B \in \mathrm{GL}_{n}(\mathbb{R}), \operatorname{det}(A) \operatorname{det}(B)=1\right\} .
$$

The product map $H^{-} \times H^{0} \times H \rightarrow G$ is a local diffeomorphism; we will choose $r_{0}$ so that the inverse of this map is well defined on $B_{G}\left(r_{0}\right)$. Note that $H^{-}$is expanding horospherical with respect to $g_{-t}, t>0$, while $H^{0}$ is centralized by $\left\{g_{t}\right\}$. Thus, the inner automorphism $\Phi_{t}$ of $G$ given by $\Phi_{t}(g) \stackrel{\text { def }}{=} g_{t} h\left(g_{t}\right)^{-1}$ is non-expanding on the group

$$
\tilde{H} \stackrel{\text { def }}{=} H^{-} H^{0}=\left\{\left(\begin{array}{cc}
A & 0 \\
Y & B
\end{array}\right)\right\} ;
$$

in fact, one has

$$
\forall r>0 \forall t>0 \quad \Phi_{t}\left(B_{\tilde{H}}(r)\right) \subset B_{\tilde{H}}(r)
$$

Let us choose Haar measures $\nu^{-}, \nu^{0}$ on $H^{-}, H^{0}$ respectively, normalized so that $\mu$ is locally almost the product of $\nu^{-}, \nu^{0}$ and $\nu$. By the latter, in view of [B, Ch. VII, $\S 9$, Proposition 13], we mean that $\mu$ can be expressed via $\nu^{-}, \nu^{0}$ and $\nu$ in the following way: for any $\varphi \in L^{1}(G)$

$$
\int_{H^{-} H^{0} H} \varphi(g) d \mu(g)=\int_{H^{-} \times H^{0} \times H} \varphi\left(h^{-} h^{0} h\right) \Delta\left(h^{0}\right) d \nu^{-}\left(h^{-}\right) d \nu^{0}\left(h^{0}\right) d \nu(h),
$$

where $\Delta$ is the modular function of (the non-unimodular group) $\tilde{H}$.

The 'thickening' will be based on the following properties of the Sobolev norm, cf. [KM1, Lemma 2.4.7]: 
Lemma 2.2. (a) For any $r>0$, there exists a nonnegative function $\theta \in C_{\text {comp }}^{\infty}\left(\mathbb{R}^{N}\right)$ such that $\operatorname{supp}(\theta)$ is inside $B(r), \int_{\mathbb{R}^{N}} \theta=1$, and $\|\theta\|_{\ell} \ll r^{-(\ell+N / 2)}$.

(b) Given $\theta_{1} \in C_{\text {comp }}^{\infty}\left(\mathbb{R}^{N}\right), \theta_{2} \in C_{\text {comp }}^{\infty}\left(\mathbb{R}^{N}\right)$, define $\theta \in C_{\text {comp }}^{\infty}\left(\mathbb{R}^{N}\right)$ by $\theta(x)=\theta_{1}(x) \theta_{2}(x)$. Then $\|\theta\|_{\ell} \ll\left\|\theta_{1}\right\|_{\ell}\left\|\theta_{2}\right\|_{C^{\ell}}$.

(c) Given $\theta_{1} \in C_{\text {comp }}^{\infty}\left(\mathbb{R}^{N_{1}}\right), \theta_{2} \in C_{\text {comp }}^{\infty}\left(\mathbb{R}^{N_{2}}\right)$, define $\theta \in C_{\text {comp }}^{\infty}\left(\mathbb{R}^{N_{1}+N_{2}}\right)$ by $\theta\left(x_{1}, x_{2}\right)=\theta_{1}\left(x_{1}\right) \theta_{2}\left(x_{2}\right)$. Then $\|\theta\|_{\ell} \ll\left\|\theta_{1}\right\|_{\ell}\left\|\theta_{2}\right\|_{\ell}$.

We will apply the above lemma to functions supported on small enough balls centered at identity elements in $G, H, H^{0}, H^{-}$.

Theorem 2.3. Let $f \in C_{\text {comp }}^{\infty}(H), 0<r<r_{0} / 2$ and $z \in X$ be such that

(i) $\operatorname{supp} f \subset B_{H}(r)$, and

(ii) $\pi_{z}$ is injective on $B_{G}(2 r)$.

Then for any $\psi \in W^{2, \infty}(X) \cap \operatorname{Lip}(X)$ with $\int_{X} \psi=0$ there exists $E=E(\psi)$ such that for any $t \geq 0$ one has

$$
\left|I_{f, \psi}\left(g_{t}, z\right)\right| \leq E\left(r \int_{H}|f|+r^{-(2 \ell+N / 2)}\|f\|_{\ell} e^{-\gamma t}\right),
$$

where $\gamma$ and $\ell$ are as in Theorem 2.1 and $N=m^{2}+m n+n^{2}-1=\operatorname{dim} \tilde{H}$.

Proof. Using Lemma 2.2, one can choose nonnegative functions $\theta^{-} \in C_{\text {comp }}^{\infty}\left(H^{-}\right)$, $\theta^{0} \in C_{\text {comp }}^{\infty}\left(H^{0}\right)$ with

$$
\int_{H^{-}} \theta^{-}=\int_{H^{0}} \theta^{0}=1
$$

such that

$$
\operatorname{supp}\left(\theta^{-}\right) \cdot \operatorname{supp}\left(\theta^{0}\right) \subset B_{\tilde{H}}(r)
$$

and at the same time

$$
\|\tilde{\theta}\|_{\ell} \ll r^{(2 \ell+N / 2)},
$$

where $\tilde{\theta} \in C_{\text {comp }}^{\infty}(\tilde{H})$ is defined by

$$
\tilde{\theta}\left(h^{-} h^{0}\right) \stackrel{\text { def }}{=} \theta^{-}\left(h^{-}\right) \theta^{0}\left(h^{0}\right) \Delta\left(h^{0}\right)^{-1} .
$$

Also define $\varphi \in C_{\text {comp }}^{\infty}(X)$ by $\varphi\left(h^{-} h^{0} h z\right)=\tilde{\theta}\left(h^{-} h^{0}\right) f(h)$; the definition makes sense because of (2.6) and assumptions (i), (ii) of the theorem. Then $I_{f, \psi}\left(g_{t}, z\right)$ can be reasonably well approximated by $\left\langle g_{t} \varphi, \psi\right\rangle=\left\langle\varphi, g_{-t} \psi\right\rangle$ :

$$
\begin{aligned}
& \left|I_{f, \psi}\left(g_{t}, z\right)-\left\langle\varphi, g_{-t} \psi\right\rangle\right| \\
& \underset{(2.3)}{=}\left|\int_{H} f(h) \psi\left(g_{t} h x\right) d \nu(h)-\int_{G} \tilde{\theta}\left(h^{-} h^{0}\right) f(h) \psi\left(g_{t} h^{-} h^{0} h x\right) d \mu\left(h^{-} h^{0} h\right)\right| \\
& \underset{(2.5)}{=}\left|\int_{G} \theta^{-}\left(h^{-}\right) \theta^{0}\left(h^{0}\right) f(h)\left(\psi\left(g_{t} h x\right)-\psi\left(\Phi_{t}\left(h^{-} h^{0}\right) g_{t} h x\right)\right) \Delta\left(h^{0}\right)^{-1} d \mu\left(h^{-} h^{0} h\right)\right| \\
& \underset{(2.2),(2.6)}{\leq} \sup _{g \in B_{\tilde{H}}(r), y \in X}|\psi(g y)-\psi(y)| \int_{G}\left|\theta^{-}\left(h^{-}\right) \theta^{0}\left(h^{0}\right) f(h) \Delta\left(h^{0}\right)^{-1}\right| d \mu\left(h^{-} h^{0} h\right) \\
& \underset{(2.3)}{\leq}\|\psi\|_{\text {Lip }} \cdot r \cdot \int_{H}|f| .
\end{aligned}
$$


On the other hand, in view of Lemma 2.2 and $\pi_{z}$ being a local isometry,

$$
\|\varphi\|_{\ell}=\|\tilde{\theta} \cdot f\|_{\ell} \ll\|\tilde{\theta}\|_{\ell}\|f\|_{\ell} \underset{(2.7)}{\ll} r^{-(2 \ell+N / 2)}\|f\|_{\ell},
$$

hence by $(2.1)$

$$
\left|\left\langle g_{t} \varphi, \psi\right\rangle\right| \ll r^{(2 \ell+N / 2)}\|f\|_{\ell}\|\psi\|_{\ell} e^{-\gamma t},
$$

finishing the proof.

Remark 2.4. In order to derive Theorem 1.1 from Theorem 2.3 it suffices to choose $r=e^{-\beta t}$ for some suitable $\beta$. The same trick will help us in the proof of Theorem 1.3. Note that $t$ needs to be taken large enough so that condition (ii) of Theorem 2.3 is satisfied for all $z \in L$. The latter is possible because, in view of the compactness of $L$ and discreteness of $\Gamma$ in $G$, the value

$$
r(L) \stackrel{\text { def }}{=} \inf _{z \in L} \sup \left\{r>0 \mid \pi_{z}: G \rightarrow X \text { is injective on } B(r)\right\}
$$

is positive; we will call it the injectivity radius of $L$.

Remark 2.5. It is worthwhile to point out that $H$ being the expanding horospherical subgroup relative to $g_{t}, t>0$, was crucially important for the proof. When $g_{t}$ is replaced with $g_{\mathbf{t}}$ where $\mathbf{t}$ is an arbitrary element of $\mathfrak{a}^{+}$, one can still talk about $\Phi_{\mathbf{t}}$, the inner automorphism of $H$ given by

$$
\Phi_{\mathbf{t}}(h) \stackrel{\text { def }}{=} g_{\mathbf{t}} h\left(g_{\mathbf{t}}\right)^{-1} .
$$

It is expanding on $H$, since the latter is contained in the expanding horospherical subgroup relative to $g_{\mathbf{t}}$; however it is not non-expanding on $\tilde{H}$ in the sense of $(2.2)$, thus there is no guarantee that $I_{f, \psi}\left(g_{t}, z\right)$ is close to $\left\langle\varphi, g_{-t} \psi\right\rangle$ for $\varphi$ constructed as in the above proof. We bypass this difficulty by means of an additional step, based on the nondivergence phenomenon, to be described in the next section.

\section{Quantitative nondivergence}

For any $\varepsilon>0$ consider

$$
K_{\varepsilon} \stackrel{\text { def }}{=} \pi\left(\left\{g \in G \mid\|g \mathbf{v}\| \geq \varepsilon \quad \forall \mathbf{v} \in \mathbb{Z}^{k} \backslash\{0\}\right\}\right) .
$$

In other words, $K_{\varepsilon}$ consists of lattices in $\mathbb{R}^{k}$ with no nonzero vector of length less than $\varepsilon$. These sets are compact by virtue of Mahler's Compactness Criterion (see $[\mathrm{R}$, Corollary 10.9$]$ or $[\mathrm{BM}])$. Here $\|\cdot\|$ can be any norm on $\mathbb{R}^{k}$ which we will from now on take to be the standard Euclidean norm.

It was proved in [KM2], refining previous work on non-divergence of unipotent flows [M, D], that certain polynomial maps from balls in Euclidean spaces to $X$ cannot take values outside of $K_{\varepsilon}$ on a set of big measure. Namely, the following is a special case of [BKM, Theorem 6.2] (see also [KLW, KT, K] for further generalizations): 
Theorem 3.1. For $d \in \mathbb{N}$, let $\varphi$ be a map $\mathbb{R}^{d} \rightarrow \mathrm{GL}_{k}(\mathbb{R})$ such that

(i) all coordinates (matrix elements) of $\varphi(\cdot)$ are affine (degree 1 polynomials), and let a ball $B \subset \mathbb{R}^{d}$ and $0<\rho \leq 1$ be such that

(ii) for any $j=1, \ldots, k-1$ and any $\mathbf{w} \in \bigwedge^{j}\left(\mathbb{Z}^{k}\right) \backslash\{0\}$ one has

$$
\|\varphi(x) \mathbf{w}\| \geq \rho \quad \text { for some } x \in B .
$$

Then for any positive $\varepsilon \leq \rho$ one has

$$
\lambda\left(\left\{x \in B \mid \pi(\varphi(x)) \notin K_{\varepsilon}\right\}\right) \ll\left(\frac{\varepsilon}{\rho}\right)^{1 / d(k-1)} \lambda(B) .
$$

Here $\lambda$ is Lebesgue measure on $\mathbb{R}^{d}$, and the Euclidean ${ }^{1}$ norm $\|\cdot\|$ is naturally extended from $\mathbb{R}^{k}$ to its exterior powers. We remark that the way assumption (i) is used in the proof is by verifying that all the functions $x \mapsto\|\varphi(x) \mathbf{w}\|$, where $\mathbf{w} \in \bigwedge^{j}\left(\mathbb{Z}^{k}\right)$, are $(C, \alpha)$-good on $\mathbb{R}^{d}$, with some fixed $C=C(d, k)>0$ and $\alpha=$ $1 / d(k-1)$, the exponent appearing in (3.1). See [KM2] for more detail.

Our plan is to apply Theorem 3.1 with $\varphi: M_{m, n} \rightarrow G$ given by

$$
\varphi(Y)=g_{\mathbf{t}} u_{Y} g
$$

for some $g \in G$ and $\mathbf{t} \in \mathfrak{a}^{+}$. It is clear that assumption (i) holds. As for (ii), we will need to have uniformity in $\mathbf{t} \in \mathfrak{a}^{+}$and in $g$ such that $\pi(g)$ belongs to a compact subset of $X$. This can be extracted from the next lemma, which is immediate from [KW, Proposition 2.4] applied to the representations of $G$ on $\bigwedge^{j}\left(\mathbb{R}^{k}\right), j=$ $1, \ldots, k-1$ :

Lemma 3.2. There exists $\alpha>0$ with the following property. Let $B$ be a ball centered at 0 in $M_{m, n}$. Then one can find $b>0$ such that for any $j=1, \ldots, k-1$, any $\mathbf{w} \in \bigwedge^{j}\left(\mathbb{R}^{k}\right)$ and any $\mathbf{t} \in \mathfrak{a}^{+}$one has

$$
\sup _{Y \in B}\left\|g_{\mathbf{t}} u_{Y} \mathbf{w}\right\| \geq b e^{\alpha\lfloor\mathbf{t}\rfloor}\|\mathbf{w}\| .
$$

Corollary 3.3. Let $B$ be a neighborhood of 0 in $M_{m, n}$ and let $L \subset X$ be compact. Then there exists $b>0$ such that for any $j=1, \ldots, k-1$, any $\mathbf{w} \in \bigwedge^{j}\left(\mathbb{Z}^{k}\right) \backslash\{0\}$, any $g \in \pi^{-1}(L)$ and any $\mathbf{t} \in \mathfrak{a}^{+}$one has

$$
\sup _{Y \in B}\left\|g_{\mathbf{t}} u_{Y} g \mathbf{w}\right\| \geq b e^{\alpha\lfloor\mathbf{t}\rfloor} .
$$

Proof. Apply the above lemma with $\mathbf{w}$ replaced by $g \mathbf{w}$; it follows from the compactness of $L$ and discreteness of $\bigwedge^{j}\left(\mathbb{Z}^{k}\right)$ in $\bigwedge^{j}\left(\mathbb{R}^{k}\right)$ that

$$
\inf \left\{\|g \mathbf{w}\| \mid \pi(g) \in L, \mathbf{w} \in \bigwedge^{j}\left(\mathbb{Z}^{k}\right) \backslash\{0\}, j=1, \ldots, k-1\right\}
$$

is positive.

\footnotetext{
${ }^{1}$ In [KM2] the statement of Theorem 5.2 involved the sup norm instead of the Euclidean one, which resulted in a restriction for $\rho$ to be not greater than $1 / k$; thus we chose to refer to [BKM] for the Euclidean norm version.
} 
Corollary 3.4. Let $L \subset X$ be compact and let $B \subset H$ be a ball centered at $e \subset H$. Then there exists $T=T(B, L)$ such that for every $0<\varepsilon<1$, any $z \in L$ and any $\mathbf{t} \in \mathfrak{a}^{+}$with $\lfloor\mathbf{t}\rfloor \geq T$ one has

$$
\nu\left(\left\{h \in B \mid g_{\mathbf{t}} h z \notin K_{\varepsilon}\right\}\right) \ll \varepsilon^{\frac{1}{m n(k-1)}} \nu(B) .
$$

Proof. Define $T$ by $b e^{\alpha T}=1$, where $\alpha$ is given by Lemma 3.2 and $b$ by Corollary 3.3 applied to $\log (B) \subset M_{m, n}$ and $L$. (Note that the exponential map from $M_{m, n}$ to $H$ is an isometry.) Take $\varphi$ as in (3.2) with $g \in \pi^{-1}(L)$. Then, in view of Corollary 3.3 , assumption (ii) of Theorem 3.1, with $d=m n$, will be satisfied with $\rho=1$ as long as $\lfloor\mathbf{t}\rfloor \geq T$.

We conclude this section by an estimate of the injectivity radius of $K_{\varepsilon}$, to make it possible to combine the above corollary with Theorem 2.3. Observe that any lattice $\Lambda \in K_{\varepsilon}$ can be generated by vectors of norm $\ll 1 / \varepsilon^{k-1}$; if $g \Lambda=\Lambda$ and $g \neq e$, then for one of those vectors $\mathbf{v}$ one has $\|g \mathbf{v}-\mathbf{v}\| \geq \varepsilon$. This implies that $\operatorname{dist}(e, g) \gg \varepsilon^{k}$. We arrive at

Proposition 3.5. There exists positive $c=c(k)$ such that $r\left(K_{\varepsilon}\right) \geq c \cdot \varepsilon^{k} \forall \varepsilon>0$.

\section{Proof of Theorem 1.3}

Our goal in this section will be to find $\tilde{\gamma}>0$ such that for any $f \in C_{\text {comp }}^{\infty}(H)$, $\psi \in W^{2, \infty}(X) \cap \operatorname{Lip}(X)$ with $\int_{X} \psi=0$ and compact $L \subset X$ there exists $\tilde{C}>0$ such that for all $z \in L$ and all $\mathbf{t} \in \mathfrak{a}^{+}$one has

$$
\left|I_{f, \psi}\left(g_{\mathbf{t}}, z\right)\right| \leq \tilde{C} e^{-\tilde{\gamma}\lfloor\mathbf{t}\rfloor} .
$$

Then Theorem 1.3 will follow by applying (4.1) with $\psi$ replaced by $\psi-\int_{X} \psi$. Note also that, by increasing $\tilde{C}$ if needed, it is enough to prove (4.1) for $\mathbf{t}$ with large enough $\lfloor\mathbf{t}\rfloor$.

Given $\mathbf{t} \in \mathfrak{a}^{+}$, define $t \stackrel{\text { def }}{=}\lfloor\mathbf{t}\rfloor / 2$, and let

$$
\mathbf{u}=\mathbf{u}(\mathbf{t}) \stackrel{\text { def }}{=} \mathbf{t}-\left(\frac{t}{m}, \ldots, \frac{t}{m}, \frac{t}{n}, \ldots, \frac{t}{n}\right) .
$$

Note that $\mathbf{u} \in \mathfrak{a}^{+},\lfloor\mathbf{u}\rfloor \geq\lfloor\mathbf{t}\rfloor / 2=t$, and $g_{\mathbf{t}}=g_{t} g_{\mathbf{u}}$ (here $g_{\mathbf{t}}$ and $g_{\mathbf{u}}$ are defined via (1.4), and $g_{t}$ is as in (1.2)).

Take a function $\theta$ supported on $B_{H}(r)$ as in Lemma 2.2(a), with $r=e^{-\beta t}$ where $\beta$ is to be specified later; since $\int_{H} \theta=1$ and $\nu$ is translation-invariant, one can write

$$
\begin{aligned}
I_{f, \psi}\left(g_{\mathbf{t}}, z\right) & =\int_{H} f(h) \psi\left(g_{\mathbf{t}} h z\right) d \nu(h) \int_{H} \theta(y) d \nu(y) \\
& =\int_{H} \int_{H} f\left(\Phi_{\mathbf{u}}^{-1}(y) h\right) \theta(y) \psi\left(g_{t} g_{\mathbf{u}} \Phi_{\mathbf{u}}^{-1}(y) h z\right) d \nu(y) d \nu(h) \\
& =\int_{H} \int_{H} f\left(\Phi_{\mathbf{u}}^{-1}(y) h\right) \theta(y) \psi\left(g_{t} y g_{\mathbf{u}} h z\right) d \nu(y) d \nu(h) .
\end{aligned}
$$

Note that $\Phi_{\mathbf{u}}^{-1}$ is a contracting automorphism of $H$, in fact, one has

$$
\operatorname{dist}\left(e, \Phi_{\mathbf{u}}^{-1}(h)\right) \leq e^{-2\lfloor\mathbf{u}\rfloor} \operatorname{dist}(e, h) \leq e^{-2 t} \operatorname{dist}(e, h)
$$


for any $h \in H$. Choose $B=B(r)$ containing supp $f$. Then the supports of all functions of the form $h \mapsto f\left(\Phi_{\mathbf{u}}^{-1}(y) h\right)$ are contained in

$$
\tilde{B} \stackrel{\text { def }}{=} B\left(r+e^{-(2+\beta) t}\right) .
$$

By taking $t$ large enough it is safe to assume that

$$
e^{-\beta t}<r_{0} / 2,
$$

$\nu(\tilde{B}) \leq 2 \nu(B)$, and also that $t>T \stackrel{\text { def }}{=} T(\tilde{B}, L)$ as in Corollary 3.4. Now define $\varepsilon$ by

$$
\varepsilon=\left(\frac{2}{c} e^{-\beta t}\right)^{1 / k},
$$

where $c$ is from Proposition 3.5, and denote

$$
A \stackrel{\text { def }}{=}\left\{h \in \tilde{B} \mid g_{\mathbf{u}} h z \notin K_{\varepsilon}\right\} .
$$

Then for any $\mathbf{u} \in \mathfrak{a}^{+}$with $\lfloor\mathbf{u}\rfloor \geq T$ and any $z \in L$ one knows, in view of Corollary 3.4 , that

$$
\nu(A) \ll \varepsilon^{\frac{1}{m n(k-1)}} \nu(\tilde{B}) .
$$

Hence the absolute value of

$$
\int_{A} \int_{H} f\left(\Phi_{\mathbf{u}}^{-1}(y) h\right) \theta(y) \psi\left(g_{t} y g_{\mathbf{u}} h z\right) d \nu(y) d \nu(h)
$$

is

$$
\ll \varepsilon^{\frac{1}{m n(k-1)}} \nu(\tilde{B}) \sup |f| \sup |\psi| \int_{H} \theta \ll \sup |f| \sup |\psi| \nu(B) \cdot e^{-\frac{\beta}{m n k(k-1)} t} .
$$

Now let us assume that $h \in \tilde{B} \backslash A$, and apply Theorem 2.3 with $r=e^{-\beta t}, g_{\mathbf{u}} h z$ in place of $z$ and

$$
f_{h}(y) \stackrel{\text { def }}{=} f\left(\Phi_{\mathbf{u}}^{-1}(y) h\right) \theta(y)
$$

in place of $f$. Clearly condition (i) follows from (4.3), and, since $g_{\mathbf{u}} h z \in K_{\varepsilon}$ whenever $h \notin A$, condition (ii) is satisfied in view of Proposition 3.5 and (4.4). Also, because $\left.\Phi_{\mathbf{u}}^{-1}\right|_{H}$ is contracting, partial derivatives of $f$ will not increase after precomposition with $\Phi_{\mathbf{u}}^{-1}$, and thus

$$
\left\|f_{h}\right\|_{\ell} \underset{\text { Lemma 2.2(b) }}{\ll}\|f\|_{C^{\ell}}\|\theta\|_{\ell} \underset{\text { Lemma 2.2(a) }}{\ll} r^{-(\ell+m n / 2)}\|f\|_{C^{\ell}} .
$$

This way one gets:

$$
\begin{array}{r}
\left|\int_{\tilde{B} \backslash A} \int_{H} f\left(\Phi_{\mathbf{u}}^{-1}(y) h\right) \theta(y) \psi\left(g_{t} y g_{\mathbf{u}} h z\right) d \nu(y) d \nu(h)\right| \leq \int_{\tilde{B} \backslash A}\left|I_{f_{h}, \psi}\left(g_{t}, g_{\mathbf{u}} h z\right)\right| d \nu(h) \\
\leq \underset{(2.4)}{\leq} E(\psi)\left(r \int_{H}\left|f_{h}\right|+r^{-(2 \ell+N / 2)}\left\|f_{h}\right\|_{\ell} e^{-\gamma t}\right) \nu(\tilde{B}) \\
\underset{(4.5)}{\ll} E(\psi)\left(\sup |f| \cdot e^{-\beta t}+\|f\|_{\left.C^{\ell} \cdot e^{-(\gamma-(2 \ell+N / 2) \beta) t}\right) \nu(B) .}\right.
\end{array}
$$


Combaning the two estimates above, one can conclude that

$$
\begin{aligned}
\left|I_{f, \psi}\left(g_{\mathbf{t}}, z\right)\right| & \ll C_{1} e^{-\frac{\beta}{m n k(k-1)} t}+C_{2} e^{-\beta t}+C_{3} e^{-(\gamma-(2 \ell+N / 2) \beta) t} \\
& \leq \max \left(C_{1}, C_{2}\right) e^{-\frac{\beta}{m n k(k-1)} t}+C_{3} e^{-(\gamma-(2 \ell+N / 2) \beta) t},
\end{aligned}
$$

where $C_{i}, i=1,2,3$, depend on $f, \psi$ and $L$. An elementary computation shows that choosing $\beta$ equalizing the two exponents above will produce

$$
\tilde{\gamma}=\frac{\gamma}{1+m n k(k-1)(2 \ell+N / 2)}
$$

such that (4.1) will hold with $\tilde{C} \ll \max \left(C_{1}, C_{2}, C_{3}\right)$.

\section{REFERENCES}

[B] N. Bourbaki, Éléments de mathematique, Livre VI: Integration, Chapitre 7: Mesure de Haar, Chapitre 8: Convolution et representations, Hermann, Paris, 1963.

[BKM] V. Bernik, D. Kleinbock, and G. A. Margulis, Khintchine-type theorems on manifolds: the convergence case for standard and multiplicative versions, Internat. Math. Res. Notices (2001), no. 9, 453-486.

[BM] B. Bekka and M. Mayer, Ergodic theory and topological dynamics of group actions on homogeneous spaces, Cambridge University Press, Cambridge, 2000.

[D] S. G. Dani, On orbits of unipotent flows on homogeneous spaces, II, Ergodic Theory Dynamical Systems 6 (1986), 167-182.

[K] D. Kleinbock, An extension of quantitative nondivergence and applications to Diophantine exponents, Trans. Amer. Math. Soc. 360 (2008), 6497-6523.

$[\mathrm{KLW}]$ D. Kleinbock, E. Lindenstrauss and B. Weiss, On fractal measures and Diophantine approximation, Selecta Math. 10 (2004), no. 4, 479-523.

[KM1] D. Kleinbock and G. A. Margulis, Bounded orbits of nonquasiunipotent flows on homogeneous spaces, Amer. Math. Soc. Transl. 171 (1996), 141-172.

[KM2] Flows on homogeneous spaces and Diophantine approximation on manifolds, Ann. Math. 148 (1998), 339-360.

[KM3] Logarithm laws for flows on homogeneous spaces, Inv. Math. 138 (1999), 451-494.

$[\mathrm{KT}]$ D. Kleinbock and G. Tomanov, Flows on S-arithmetic homogeneous spaces and applications to metric Diophantine approximation, Comm. Math. Helv. 82 (2007), 519-558.

$[\mathrm{KW}]$ D. Kleinbock and B. Weiss, Dirichlet's theorem on diophantine approximation and homogeneous flows, J. Mod. Dyn. 2 (2008), 43-62.

[M] G. A. Margulis, On the action of unipotent group in the space of lattices, In: Proceedings of the Summer School on group representations, (Budapest 1971), Académiai Kiado, Budapest, 1975, pp. 365-370.

[R] M. S. Raghunathan, Discrete subgroups of Lie groups, Springer-Verlag, Berlin and New York, 1972.

[S] N. A. Shah, Equidistribution of expanding translates of curves and Dirichlet's theorem on diophantine approximation, Invent. Math. 177, no. 3, 509-532.

Department of Mathematics, Brandeis University, Waltham, MA 02454

E-mail address: kleinboc@brandeis.edu

Department of Mathematics, Yale University, New Haven, CT 06520

E-mail address: margulis@math.yale.edu 